\title{
Numerical Analysis of a Diffusion Flame Under Water Mist Jet Influence
}

\author{
M. De la Cruz-Ávila ${ }^{1}$, E. Martínez-Espinosa² ${ }^{2}$ G. Polupan ${ }^{1}$ \\ ${ }^{1}$ Instituto Politécnico Nacional, LABINTHAP, ESIME, CDMX, \\ Mexico \\ ${ }^{2}$ UNAM Ciudad Universitaria, Instituto de Ingeniería, CDMX, \\ Mexico \\ mauriciodlca1@gmail.com
}

\begin{abstract}
A water mist jet for a steam generator of direct contact with a diffusion flame is numerically analyzed in this paper. Numerical simulation is conducted for an 8 Lug-Bolt array in a confined system. The diffusion flame is modelled in the Eulerian - Eulerian multiphase approach where the reaction species are oxygen which is injected by a $16.9 \mathrm{~mm}$ central nozzle, methane by four peripheral $5.86 \mathrm{~mm}$ nozzles as the water mist and with a droplet size of $10 \mu \mathrm{m}$. Numerical simulations are developed with the Reynolds Averaged Navier-Stokes and the Realizable $k-\varepsilon$ turbulence model is considered. The Eddy Dissipation Model is implemented in order to calculate the effect of turbulent chemical reaction rate. Predictions show some instabilities that are located within the flame structure as long as the fuel moisture percentage is less than $1 \%$. If the mist fraction for vaporization increases, the instabilities do not affect the internal flame structure but instabilities becomes relevant to the end of the calculus domain. The decrement about $60 \%$ of the water mist velocity due to a vapor gap around the micro droplets is the mean cause of the instabilities. Therefore, a very precise control of the numerical parameters is so important for multiphase approximations.
\end{abstract}

Keywords: Eulerian - Eulerian multiphase approach, multiphase combustion, water mist injection, direct vaporization.

\section{Introduction}

In the open literature there are many studies focused on two gas streams, which consider an annular fuel and oxidizer injection shape. For example, the study carried out by Fossa [1], and Cioncollini [2] analyze two-phase mixture flows. Grech [3], $\mathrm{Cu}-$ trone [4], and Smith [5] conducted numerical simulations with Reynolds-Averaged Navier-Stokes (RANS) method for diffusion combustion process in jet propulsion rockets and gas turbines. Lopez-Parra and Turan [6] have simulated a methane turbulent jet diffusion flame with the Eddy Dissipation Model (EDM) [7] and the standard 
$k-\varepsilon$ turbulence model with satisfactory results. Finally, the mixing fluids process in diffusion flame systems is extremely important because the fuel and oxidizer are injected independently and combustion starts when the mixture reaches flammability limits (upper or lower) [8].

However, all this studies are focused on the flame development but not on the numerical control for a multiphase combustion-vaporization scheme. Therefore, this paper is focused on the numerical control settings for the numerical diffusion flamewater mist in a multiphase scheme approximation. The water mist injection flow for three cases are analyzed in order to compare its influence over development during the steam generation. This work allows to be applied to further numerical studies in turbulent combustion, water drops vaporization and quenching flames.

\section{Numerical Details}

Numerical simulations are conducted for 8 Lug-Bolt system where oxygen is injected in a $16.9 \mathrm{~mm}$ central nozzle, methane and water mist by four peripheral $5.86 \mathrm{~mm}$ nozzles respectively. The simulations used the standard methane, water and oxygen properties for mixture species. Jets are under atmospheric conditions of $1 \mathrm{~atm}$ and $298 \mathrm{~K}$ in a confined system as shown in Fig. 1.

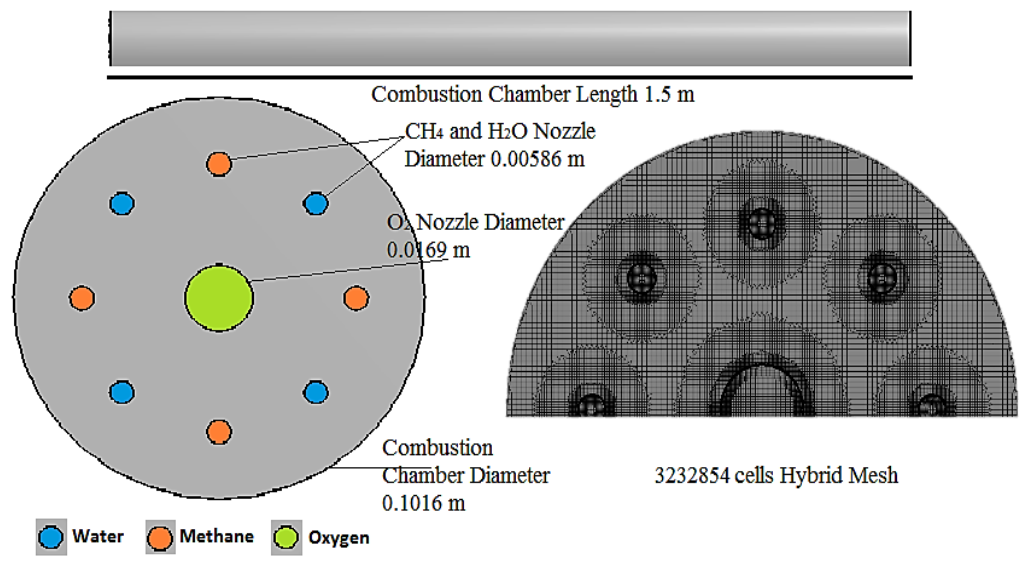

Fig. 1. Computational domain, settings and mesh.

The combustion chamber has $101.6 \mathrm{~mm}$ diameter $\left(D_{c}\right)$ and $1500 \mathrm{~mm}$ of total length (L). The aim of the numerical approximations is to analyse the influence of the water mist affecting the mixture process for three flow Cases. An Eulerian-Eulerian approach was implemented for representing multiphase phenomenon and combustion species were modelled as the continuous phase ( $p$ phase 1$)$ and the water mist as the dispersed phase ( $q$ phase 2). Simulations consider mean velocity profile of $10 \mathrm{~m} / \mathrm{s}$ for every stream. The water mist droplets are analysed for three different cases. The micro-droplet diameter remains constant with $10 \mu \mathrm{m}$ while the injection mist flow is 
$0.005,0.010$ and $0.015 \mathrm{~kg} / \mathrm{s}$. Pressure outlet to air was use for boundary outlet a nonslip and adiabatic treatment for the calculus domain wall was use. A mesh with 3232854 cells (Fig.1.), which is based on the proper resolution of the micro-droplets dynamics with a size of 10 microns and the gas phase flow coupling was used as described by Yuval Dagan et al [9]. Both phases are proposed as unreactive fluids to ensure a good tracking of properties at the interphase.

\subsection{Constitutive Equations}

In first instance, the numerical analysis of the oxygen-methane mixture process is of the physical nature. This implies that the equations to be solved are mass conservation, the momentum quantity, energy and chemical. These equations in multiphase Eulerian-Eulerian approach model solution are defined by the following expressions.

\section{Mass conservation.}

$$
\frac{\partial}{\partial \mathrm{t}}\left(\alpha_{\mathrm{q}} \overline{\rho_{\mathrm{q}}}\right)+\nabla \cdot\left(\alpha_{\mathrm{q}} \overline{\rho_{\mathrm{q}}} \overrightarrow{\mathrm{v}}_{\mathrm{q}}\right)=\sum_{\mathrm{p}=1}^{\mathrm{n}}\left(\overline{\dot{\mathrm{m}}_{\mathrm{pq}}} \overline{\dot{\mathrm{m}}_{\mathrm{qp}}}\right)+\overline{\mathrm{S}}_{\mathrm{q}},
$$

where $\alpha_{q}$ is the volume fraction, $\vec{v}_{q}$ is the velocity of phase $q$ and $\dot{m}_{p q}$ characterizes the mass transfer from the pth to qth phase, and $\dot{m}_{q p}$ characterizes the mass transfer from the phase $q$ to phase $p, \rho$ is the phase density and $S_{q}$ is the source term.

\section{Momentum.}

$$
\begin{aligned}
& \frac{\partial}{\partial \mathrm{t}}\left(\alpha_{\mathrm{q}} \overline{\rho_{\mathrm{q}}} \widetilde{\overrightarrow{\mathrm{v}}}_{\mathrm{q}}\right)+\nabla \cdot\left(\alpha_{\mathrm{q}} \overline{\rho_{\mathrm{q}}}{\overrightarrow{\vec{v}_{\mathrm{q}}}}_{\overrightarrow{\mathrm{v}}_{\mathrm{q}}}\right)=-\alpha_{\mathrm{q}} \nabla \tilde{\mathrm{p}}+\nabla \cdot \overline{\overrightarrow{\mathrm{\tau}}_{\mathrm{q}}} \overline{\rho_{\mathrm{q}}} \overline{\overrightarrow{\mathrm{g}}}+
\end{aligned}
$$

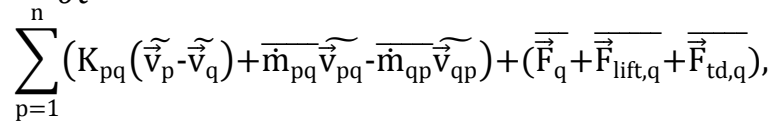

where $\left(\mathrm{K}_{p q}\left(=\mathrm{K}_{q p}\right)\right)$ is the interphase momentum exchange coefficient and $\vec{v}_{q, p}$ are the phase velocities. Note that represents the mean interphase momentum exchange and does not include any contribution due to turbulence. The turbulent interphase momentum exchange is modelled with the turbulent dispersion force term $\vec{F}_{t d, q}$ and $\vec{F}_{q}$ the external body forces which is equal to $0 . \overline{\bar{\tau}}_{q}$ is the $q^{\text {th }}$ phase stress tensor $p$ and $g$ are the pressure ante gravity respectively.

Conservation of Energy. To describe the conservation of energy in Eulerian multiphase applications, the equation can be written in terms of each phase:

$$
\begin{gathered}
\frac{\partial}{\partial \mathrm{t}}\left(\alpha_{\mathrm{q}} \overline{\rho_{\mathrm{q}}} \widetilde{\mathrm{h}_{\mathrm{q}}}\right)+\nabla \cdot\left(\alpha_{\mathrm{q}} \overline{\rho_{\mathrm{q}}} \widetilde{\overrightarrow{\mathrm{v}}_{\mathrm{q}}} \widetilde{\mathrm{h}_{\mathrm{q}}}\right)= \\
\alpha_{\mathrm{q}} \frac{\partial \overline{\mathrm{p}_{\mathrm{q}}}}{\partial \mathrm{t}}+\overline{\overrightarrow{\mathrm{\tau}}_{\mathrm{q}}}: \nabla \widetilde{\overrightarrow{\mathrm{v}}_{\mathrm{q}}}-\nabla \cdot \widetilde{\overrightarrow{\mathrm{q}}_{\mathrm{q}}}+\mathrm{S}_{\mathrm{q}}+\sum_{\mathrm{p}=1}^{\mathrm{n}}\left(\mathrm{Q}_{\mathrm{pq}}+\overline{\dot{\mathrm{m}}_{\mathrm{pq}}} \widetilde{\mathrm{h}_{\mathrm{pq}}}-\overline{\dot{\mathrm{m}}_{\mathrm{qp}}} \widetilde{\mathrm{h}_{\mathrm{qp}}}\right) .
\end{gathered}
$$

Conservation of Species. The chemical species conservation equation for a multiphase mixture can be represented in the following form: 


$$
\begin{gathered}
\frac{\partial}{\partial \mathrm{t}}\left(\overline{\rho^{\mathrm{q}}} \alpha^{\mathrm{q}} \widetilde{Y_{i}^{\mathrm{q}}}\right)+\square \cdot\left(\bar{\rho}^{\mathrm{q}} \alpha^{\mathrm{q}} \widetilde{\vec{v}^{\mathrm{q}}}{\widetilde{Y_{i}^{\mathrm{q}}}}_{i}^{\mathrm{q}}\right)= \\
-\square \cdot \alpha^{\mathrm{q}} \widetilde{\vec{J}}_{\mathrm{i}}^{\mathrm{q}}+\alpha^{\mathrm{q}} \mathrm{R}_{\mathrm{i}}^{\mathrm{q}}+\alpha^{\mathrm{q}} \mathrm{S}_{\mathrm{i}}^{\mathrm{q}}+\sum_{\mathrm{p}=1}^{\mathrm{n}}\left(\overline{\dot{m}}_{\mathrm{p}^{\mathrm{i}} \mathrm{q}^{j}} \overline{\dot{\mathrm{m}}}_{\mathrm{q}^{\mathrm{j}} \mathrm{p}^{\mathrm{i}}}\right)+\mathrm{R},
\end{gathered}
$$

where $\mathcal{R}$ is the reaction rate and $\overline{\vec{\tau}_{q}}=\overline{\rho_{q}} \overline{\vec{v}_{q} \vec{v}_{q}}-\overline{\rho_{q}} \widetilde{\vec{v}_{q}} \widetilde{\vec{v}_{q}}, \widetilde{\vec{q}_{q}}=\overline{\rho_{q}} \overline{\vec{v}_{q} h_{q}}-\overline{\rho_{q}} \widetilde{\vec{v}_{q}} \widetilde{h_{q}}$, $\widetilde{\vec{J}_{l}^{q}}=\overline{\rho^{q}} \overline{\vec{v}^{q} Y_{l}^{q}}-\overline{\rho^{q}} \widetilde{\vec{v} q} \widetilde{Y_{l}^{q}}$, are the average fluctuations of Reynolds stresses, heat fluxes and mass fluxes respectively, with the sign " "-, denoting time average and " $~$ ", denoting Favre average.

The transport momentum equation averaging result into the appearance of terms containing the average fluctuations. Turbulence model involved in this paper (the Realizable $k-\varepsilon$ model) is based on the Boussinesq hypothesis, which means, the Reynolds stress tensor must be modelled in order to close the RANS equations and be proportional to the mean deformation rate tensor. The Realizable $k$ - $\varepsilon$ turbulence model describes a two scalar transport, the turbulent kinetic energy $(k)$ and its dissipation rate $(\varepsilon)$. The model has been validated experimentally for many reactive flows simulations with satisfactory results [10-12]. Numerical simulations are developed with the alternative RANS technique and considers the Realizable $k-\varepsilon$ [13] turbulence model for the equations system closure.

\subsection{Constitutive Combustion Kinetics and Combustion Modelling}

The energy production by the methane combustion has been well established by the next overall reaction:

$$
\mathrm{CH}_{4}+2 \mathrm{O}_{2}=\mathrm{CO}_{2}+2 \mathrm{H}_{2} \mathrm{O}, \Delta \mathrm{H}_{298}=-802.7 \mathrm{~kJ} / \mathrm{mol} \text {. }
$$

This overall equation is a gross simplification by the actual reaction mechanism, which involves free radical chain reactions. The numerical simulation considers a stoichiometric reaction neglecting all other subsequent reaction in the chain reaction mechanism. Nevertheless, this work main purpose is not to analyze the secondary chemical reactions. For this reason, a single-step irreversible chemical reaction was used in order to redirect computational resources to the flow development.

The species are characterized through the involved mass fractions $Y_{i}$ for $i=1$ to $N$, where $N$ is the number of species in the reacting mixture. The mass fractions $Y_{i}$ are defined by:

$$
Y_{i}=\frac{m_{i}}{m}
$$

where $m_{i}$ is the mass of species $i$ present in a given volume $V$ and $m$ is the total mass of gas in the volume. The numerical study of turbulent-reactive flows depends upon the combustion model adequate selection. The high non-linear production term for the species' conservation equation closure is one of the most challenging aspects when modeling turbulent combustion. The Eddy Dissipation Model (EDM) is used to calculate the turbulent chemical reaction rate effect. The EDM is based on the infinitely 
fast chemistry hypothesis and assumes that the reaction rate is controlled by the turbulent mixing [14]. A generalized formulation of the EDM has been proposed in order to take into account finite-rate chemistry effects. A stoichiometric relation describing chemical reactions of arbitrary complexity can be represented by the $r^{\text {th }}$ reaction equation [15]. The turbulent mixing rate is related to the turbulent eddies timescale present in the flow. The timescale used for this purpose is the so-called eddy lifetime, $\tau=k / \varepsilon$, with $k$ being the turbulent kinetic energy, $\varepsilon$ the turbulent dissipation rate and the chemistry typically described by relatively simple single or two-step mechanism. The species $i$ production net-rate due to reaction $r, R_{i, r}$ is given by the smaller (limitingvalue) of the two expression below.

\section{Base on reactants mass fraction:}

$$
\mathrm{R}_{\mathrm{i}, \mathrm{r}}=\mathrm{v}_{\mathrm{i}, \mathrm{r}}^{\prime} \mathrm{M}_{\mathrm{w}, \mathrm{i}} \mathrm{A} \rho \frac{\varepsilon}{\mathrm{k}} \min _{\mathrm{R}}\left(\frac{\mathrm{Y}_{\mathrm{R}}}{\mathrm{v}_{\mathrm{R}, \mathrm{r}}^{\prime} \mathrm{M}_{\mathrm{w}, \mathrm{R}}}\right)
$$

Base on products mass fraction:

$$
\mathrm{R}_{\mathrm{i}, \mathrm{r}}=\mathrm{v}_{\mathrm{i}, \mathrm{r}} \mathrm{M}_{\mathrm{w}, \mathrm{i}} \mathrm{AB} \rho \frac{\varepsilon}{\mathrm{k}} \frac{\sum_{\mathrm{P}} \mathrm{Y}_{\mathrm{P}}}{\sum_{\mathrm{j}}^{\mathrm{N}} \mathrm{v}^{\prime \prime}{ }_{\mathrm{j}, \mathrm{r}} \mathrm{M}_{\mathrm{w}, \mathrm{j}}},
$$

where $Y_{\mathrm{p}}$ and $Y_{R}$ the species mass fraction, $A$ and $B$ are Magnussen [14] constant for

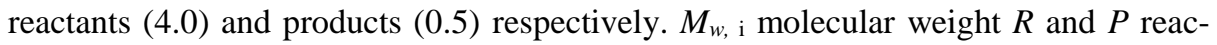
tants and products respectively.

\subsection{Numerical Fluid Reconstruction}

A 3rd order Quadratic Upstream Interpolation for Convective Kinematics (QUICK) scheme for the convective and viscous terms was considered. However, this highorder scheme is not easy to apply to unstructured grid directly. The Leonard's QUICK scheme [16] uses a quadratic fit through two upwind nodes and one downwind cell center. To find the exact location of the next upwind cell nodes would increase the geometrical complexity and consume relatively more memory and CPU time.

In a uniform grid (Fig. 2. for definitions of points WW, W, P, E, EE), the Quick scheme at the east cell-face can be written as:

$$
\begin{aligned}
& \phi_{\mathrm{e}}=\frac{1}{2}\left(\phi_{\mathrm{P}}+\phi_{\mathrm{E}}\right)-\frac{1}{8}\left(\phi_{\mathrm{w}}-2 \phi_{\mathrm{P}}+\phi_{\mathrm{E}}\right)(\mathrm{u} \geq 0), \\
& \phi_{\mathrm{e}}=\frac{1}{2}\left(\phi_{\mathrm{P}}+\phi_{\mathrm{E}}\right)-\frac{1}{8}\left(\phi_{\mathrm{EE}}-2 \phi_{\mathrm{E}}+\phi_{\mathrm{P}}\right)(\mathrm{u}<0) .
\end{aligned}
$$

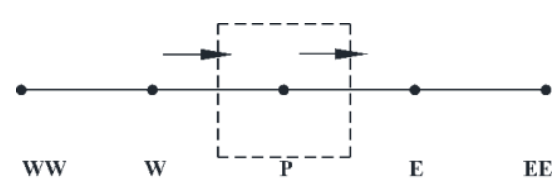

Fig. 2. QUICK scheme for a uniform grid. 
This scheme is $2^{\text {nd }}$-order accurate if the definition of the truncation error is based on approximating the spatial derivative at cell centers in the linear convection equation. Other authors $[14,15]$ have chosen alternative definitions of the truncation error, according to which QUICK becomes 3rd-order accurate. Consequently, the implementation of both specifications (mesh/scheme) provides better approximation results in comparison to those in which use is posed separately.

The $3^{\text {rd }}$-order Modified High Resolution Interface Capturing Scheme (M-HRIC) for the viscous terms. For simulations using the multiphase model, upwind schemes are generally unsuitable for interface tracking because of their overly diffusive nature. Central differencing schemes, while generally able to retain the sharpness of the interface, are unbounded and often give unphysical results. In order to overcome these deficiencies, it is better to use the modified version of the High Resolution Interface Capturing (HRIC) scheme. The modified HRIC scheme is a composite normalized variable diagram (NVD) scheme that consists of a nonlinear blend of upwind and downwind differencing. First, the normalized cell value of volume fraction, $\tilde{\phi}_{c}$ is computed and is used to find the normalized face value, $\tilde{\phi}_{f}$ as follows:

$$
\tilde{\phi}_{\mathrm{c}}=\frac{\phi_{\mathrm{D}}-\phi_{\mathrm{U}}}{\phi_{\mathrm{A}}-\phi_{\mathrm{U}}},
$$

where $\mathrm{A}$ is the acceptor cell, $\mathrm{D}$ is de donor cell, and $\mathrm{U}$ is the upwind cell and

$$
\begin{aligned}
& \tilde{\phi}_{\mathrm{f}}=\left\{\begin{array}{lr}
\tilde{\phi}_{\mathrm{c}} & \tilde{\phi}_{\mathrm{c}}<0 \text { or } \tilde{\phi}_{\mathrm{c}}>1, \\
\widetilde{2 \phi}_{\mathrm{c}} & 0 \leq \tilde{\phi}_{\mathrm{c}} \leq 0.5, \\
1 & 0.5 \leq \widetilde{\phi}_{\mathrm{c}} \leq 1 .
\end{array}\right. \\
& \begin{array}{|l|l|l|}
\hline \Phi_{\mathrm{U}} & \Phi_{\mathrm{D}} & \\
& & \Phi_{\mathrm{A}} \\
\hline
\end{array}
\end{aligned}
$$

Fig. 3. Cell Representation for Modified HRIC scheme.

\section{Results}

The heat released of the combustion process directly affect the water mist flow changing its enthalpy and as a consequence the vaporization process take places. The water vapour temperature contours for the three Cases are presented in Fig.4. In Case A, the global vapour temperature ascends to $2921 \mathrm{~K}$ because its mass flow is $0.005 \mathrm{~kg} / \mathrm{s}$ ( $50 \%$ less than case B and $75 \%$ less than $\mathrm{C}$ ) and the vaporization starts in a zone near or in the flame front. Vaporization Enthalpy direct affects the micro droplet due to its proximity size (less mas flow = lager distance between droplets). The global vapour temperature for Case B is $2489 \mathrm{~K}$ which represents a $14.7 \%$ variation respect Case A because its droplets proximity is shorter. Then, the vaporization energy needs to be higher to reach all droplet surroundings. Thus for Case $\mathrm{C}$, the global vapour temperature is $1541 \mathrm{~K}$ which represent a $47 \%$ respect Case A and $39 \%$ respect Case B. This 
result does not mean that a proportional increase water mist mass flow must be directly proportional to a temperature decrement. All this behaviour is related to the gaplap surrounding droplets and droplets proximity distance. Also, in this contours it is possible to observe vapour waves which are direct related with instabilities and associated to a density gradient at the interface when the velocity of phase $p$ and the velocity of the phase $q$ are the same $v_{p}=v_{q}$. These instabilities are exposed only at the end of the calculus domain because combustion products density are different of the water vapour and waves are present in the outer vapour structure. Results show water vapour velocity $\left(v_{q}\right)$ is equal to the combustion products velocity $\left(v_{p}\right)$ almost $60 \%$ of the injection velocity. Therefore, instabilities are more intense in case A, as shown in Fig. 4. The Fig. 5. shows droplet density contours for the maximum water mist concentration. This mist concentration is mixed with the methane stream considering it as moist fuel. When the water mist interacts with the fuel, a methane displacement occurs, thereby does not allows a correct fuel-oxygen mixture. For a water mist jet mass flow, humidity ratio and moist percent by species volume mixture is shown in Table 1. The mist concentration $\%$ represent a methane direct displacement $\%$. This percent is lower than the Upper Flammability Limit density species mixture (UFL). The flammability limits in a combustion process moist free is considered as a reference case. The reference case and moist mixture for three cases are shown in Table 1.

Table 1. Moist variation for the mixture process.

\begin{tabular}{lllllll}
\hline Case & $\begin{array}{l}\text { Moist Fuel } \\
\text { Concentration } \\
(\mathrm{kg} / \mathrm{m} 3)\end{array}$ & $\begin{array}{l}\text { Moist Mixture } \\
\text { Concentration } \\
(\mathrm{kg} / \mathrm{m} 3)\end{array}$ & Variation & $\begin{array}{l}\text { Variation } \\
\%\end{array}$ & $\begin{array}{l}\mathrm{CH} 4 \\
\text { mixture } \\
\%\end{array}$ & $\begin{array}{l}\text { O2 } \\
\text { mixture } \\
\%\end{array}$ \\
\hline Reference & 0.6558 & 0.8100 & 0 & 0 & 60.88 & 39.12 \\
$\mathrm{~A}$ & 0.6564 & 0.8198 & 0.00983 & 1.21 & 59.67 & 40.33 \\
$\mathrm{~B}$ & 0.6674 & 0.8236 & 0.01358 & 1.67 & 58.00 & 42.00 \\
$\mathrm{C}$ & 0.6732 & 0.8279 & 0.01793 & 2.21 & 55.79 & 44.21 \\
\hline
\end{tabular}

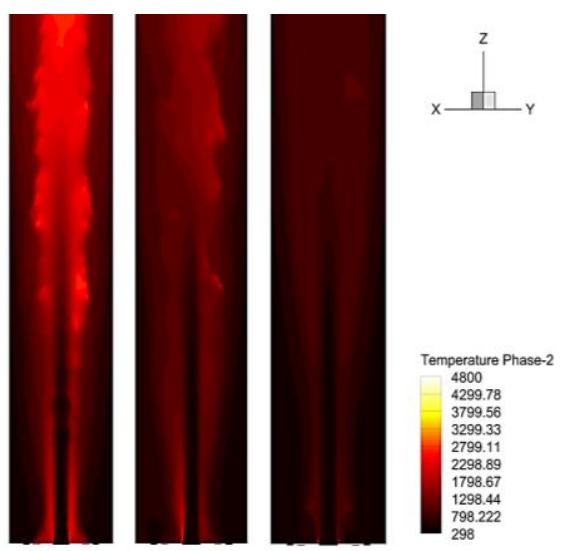

Fig. 4. Contours of water vapour Case A, B and $\mathrm{C}$ temperature $\mathrm{K}$.

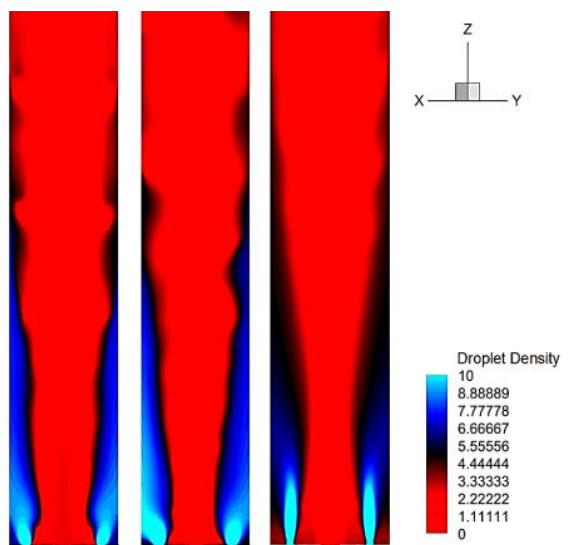

Fig. 5. Density contours Case A, Case B and Case C [dim less]. 
The fuel-oxygen mixture is produced by the turbulence induced in the methaneoxygen stream at the outlet burner and the density difference between the central jet and surroundings (mixture layer around the oxygen jet potential core). On one hand, numerical predictions show a drag effect on methane jet by the oxygen flow because the oxygen jet core has a greater amount of flow. The same behaviour is present in the three cases. Therefore, for the most relevant zone to the species mixture is located between the methane jets and oxygen stream where the flammability limits are reached. The flammability limits are represented by a methane-oxygen mixture density an optimal composition that is reached for combustion reaction. Since this global mixture have $0.8 \%$ of moist, the global density mixture increases its value in $1.21 \%$ to reach the UFL and the reaction zone is extended in the combustion chamber (represented through the iso-surfaces in Fig. 6). Whilst the reaction zone increases, the recirculation zones remain unchanged. But on the other hand, the drag effect and the recirculation also affect the water mist flow. Besides the drag effect, the velocity contraflow product of the vortex has a direct impact on the jets momentum reducing the velocity of the eight jets flow as the streamlines exhibit in Fig 7. For the three case the most affected is the case A with a water mist velocity reduction in a $60 \%$.

Considering two fluid regions, $v_{p}>v_{q}$. The fluid $p$ has uniform velocity $v_{\mathrm{p}}=10 \mathrm{~m} / \mathrm{s}$ while $q v_{q}=4 \mathrm{~m} / \mathrm{s}$ although the fluid injection $\mathrm{V}=10 \mathrm{~m} / \mathrm{s}$ for both phases. The velocity difference mentioned before is a direct consequence of the water vapour gap surrounding every droplet as mentioned by Korlie [17]. This velocity gradient causes a special offset between the mixture interfaces. It is assumed that the flow is inviscid at $\mathrm{T}_{\mathrm{pq}}<\mathrm{T}_{\mathrm{sat}}$ in this case $\mathrm{T}_{\mathrm{pq}}<\mathrm{T}_{\text {React }}$ where $\mathrm{T}_{\mathrm{pq}}$ is the mean temperature between phases and $\mathrm{T}_{\text {React }}$ is the mean exothermic reaction temperature. Therefore it can suffer a tangential velocity discontinuity at the interfacial area concentration, $A_{p^{i}}$ and $A_{p^{j}}$ just before the methane and oxygen potential cores. Those discontinuities are shown in Fig 8. Since the velocity gradient is sufficient to inflict a shear at the fluid interface, is possible to define a shear velocity or friction velocity.

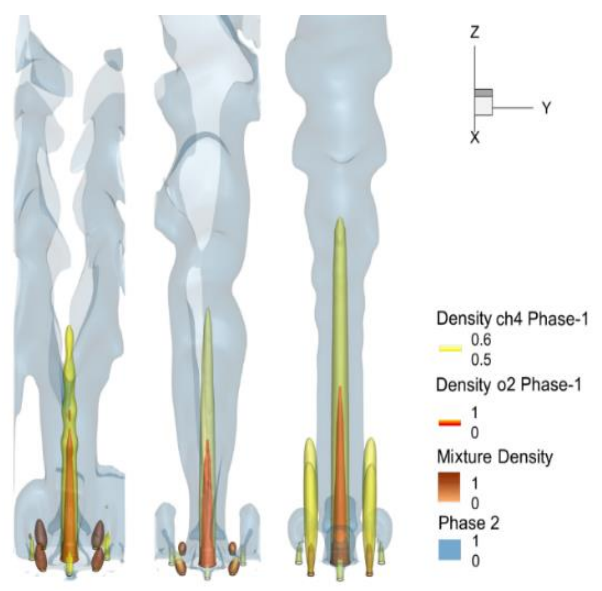

Fig. 6. Iso-surfaces of mixture components Case A, B and C.
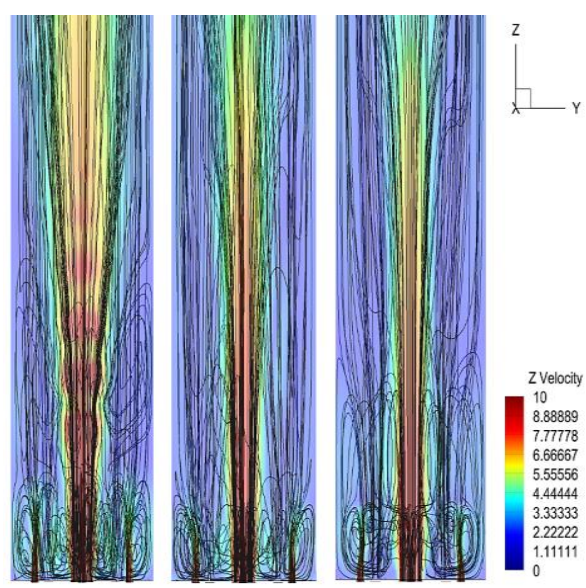

Fig. 7. Streamlines and recirculation zones Cases A, B and C. 


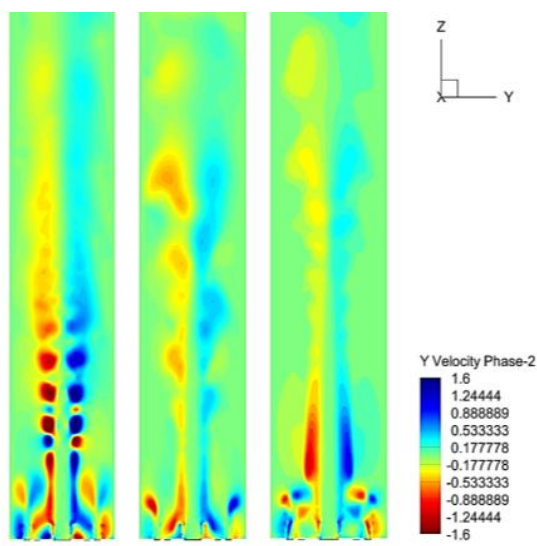

Fig. 8. Contours of tangential velocity of water mist phase $[\mathrm{m} / \mathrm{s}]$.

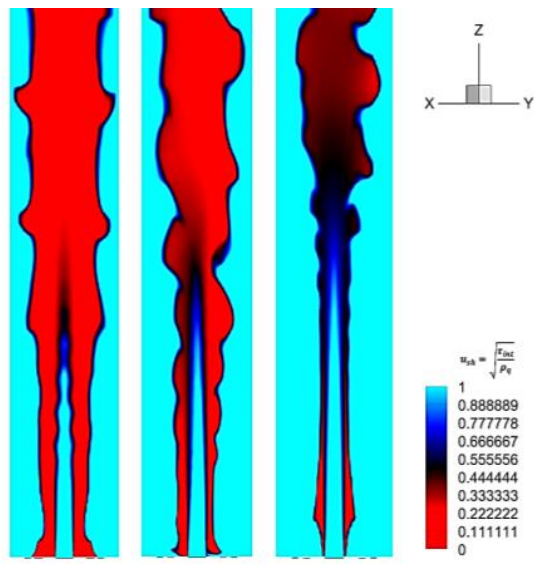

Fig. 9: Contours of $u_{s h}$ [dim less].

This kind of shear stress may be re-written in units of velocity. It is useful method to compare true velocities, such as the velocity of a mist flow in a stream and the shear between layers of flow and it is shown that in many case this velocity is $\mathrm{ush} \approx 1 / 10 v_{p}$. Before the flickering zone the outer instabilities at the outer flame layer are present. In most cases the inner instabilities are present within flame-mist layer.

The Fig. 9. demonstrates the instabilities by means of waves. The waves destabilize the structure of the oxygen jet. The outer waves destabilize the vapourcombustion products mixture structure. With the increment of mist flow the inner waves tend to diminish and the outer instabilities tends to increase.

\section{Conclusions}

The vaporization starts in a zone near the flame front and vaporization Enthalpy direct affects the micro droplet due to its proximity stablishing the relation less mass flow equal to lager distance between droplets. Then, the vaporization energy needs to be higher to reach all droplet surroundings which means a flame front temperature reduction. All this behaviour is related to the "gap-lap" surrounding droplets and droplets proximity distance. Since the mean velocity of both phases are the same $v_{p}=v_{q}$, the outer instabilities are intense by water vapour and combustion products mixing process.

Furthermore, in a fuel moisture percentage less than $1 \%$, the instabilities are conserved within the flame structure. If the vaporization mist fraction increases, the instabilities do not affect the internal structure where the methane-oxygen mixing process occurs. However, these instabilities are transferred to the outer structure of the flame by means of waves since the density variation between water vapour and combustion products is emphasized. Even the methane-oxygen reaction zone increases its value (distance) in a $68 \%$ is not a participant cause for any both kind of instabilities. The direct mechanism for these instabilities is an intensified vaporization process affecting directly the velocity of the water mist injection jets. Therefore, the vaporization effect is the leading cause of both types of instabilities. 


\section{References}

1. Fossi, M.: A Simple Model to Evaluate Direct Contact Heat Transfer and Flow Characteristics in Annular Two-Phase Flow. International Journal of heat and mass flow vol. 16, 272-279 (1995)

2. Cioncollini, A., Thome J., Lombardi C.: Algebraic Turbulence Modelling in Adiabatic Gas-Liquid Annular Two-Phase Flow. International Journal of Multiphase Flow vol. 35, pp. 580-596 (2009)

3. Grech, N., Mehdi, A., Zachos, P. K., Pachidis, V., Singh, R.: Effect of Combustor Geometry on Performance of Airblast Atomizer Under Sub-Atmospheric Conditions. Engineering Applications of Computational Fluid Mechanics vol. 6, pp. 203-213 (2012)

4. Cutrone, L., Ihme, M., Herrmann, M.: Modelling of High Pressure Mixing and Combustion in Liquid Rocket Injectors. Center for Turbulence Research, pp. 269-281 (2006)

5. Smith J. J., Schneider G., Suslov D., Oschwald M., Haidn O.: Steady-State High Pressure LOx/H2 Rocket Engine Combustion. Aerospace Science and Technology vol. 11, pp. 3947 (2007)

6. Lopez-Parra, F., Turan A.: Computational Study on the Effects of Non-Periodic Flow Perturbations on the Emissions of Soot and NOx in a Confined Turbulent Methane/Air Diffusion Flame. Combustion Science and Technology 179 (7): 1361-1384 (2007)

7. Magnussen, B. F.: On the Structure of Turbulence and a Generalized Eddy Dissipation Concept for Chemical Reaction in Turbulent Flow. AIAA Paper 1981-42 (1981)

8. De la Cruz, M., Polupan, G., Martínez, E., Carvajal, I.: Estudio Numérico del Efecto de la Presión en el Proceso de Mezcla Metano-Oxígeno en un Arreglo de Chorros 4-Lug Bolt. Información Tecnológica, CIT Chile, vol. 26, no. 2, pp. 153-162 (2015)

9. Dagan, Y., Arad, E., Tambour Y.: On the Dynamics of Spray Flames in Turbulent Flows. In: Proceedings of the Combustion Institute vol. 35, issue 2, pp. 1657-1665 (2015)

10. Vicente, W., Salinas-Vázquez, M., Martinez, E., Rodriguez, A.: Numerical Simulation of a Turbulent Lean, Premixed Combustion with an Explicit Algebraic Stress Model. J. Math. and Statistics: 1, 86-90 (2005)

11. Lin, Z. Q., Wei, F., Jin, Y.: Numerical Simulation of Pulverized Coal Combustion and No Formation. Chemical Engineering Science: 58, 5161-5171 (2003)

12. Herrmann, M.: Numerical Simulation of Turbulent Bunsen Flames with a Level Set Flamelet Model. Combustion and Flame: 145, 357-375 (2006)

13. Shih, T.-H., Liou, W. W., Shabbir, A., Yang, Z., Zhu, J.: A New k-e Eddy-Viscosity Model for High Reynolds Number Turbulent Flows Model Development and Validation. Computers and Fluids 24(3): 227-238 (1995)

14. Waterson, N.P., Deconinck, H.: A Unified Approach to the Design and Application of Bounded Higher-Order Convection Schemes. In: Proceedings of the 9th International Conference on Numerical Methods in Laminar and Turbulent Flow, Pineridge Press, Swansea, p. 203, Atlanta (1995)

15. Gaskell, P.H., Lau, A.K.C.: Curvature-Compensated Convective Transport: Smart, a New Boundedness-Preserving Transport Algorithm. International Journal for Numerical Methods in Fluids, Vol. 8, p. 617 (1988)

16. Leonard, B.P., Leschziner, M.A., McGuirk, J.: The QUICK algorithm: a uniformly 3rdorder finite-difference method for highly convective flows. In: Numerical methods in laminar and turbulent flow: proceedings of the first international conference, p. 807, Swansea, (1978)

17. Korlie, M.S.: Three-Dimensional Computer Simulation of Liquid Drop Evaporation. Computers and Mathematics with Applications 39, pp. 43-52 (2000) 